\title{
Better Correlation of Cognitive Function to White Matter Integrity than to Blood Supply in Subjects with Leukoaraiosis
}

\author{
Genlong Zhong ${ }^{1}$, Ruiting Zhang ${ }^{1}$, Yerfan Jiaerken ${ }^{2}$, Xinfeng Yu' ${ }^{2}$, Ying Zhou ${ }^{1}$, Chang Liu', \\ Longting Lin ${ }^{3}$, Lusha Tong ${ }^{1}$ and Min Lou ${ }^{1 *}$
}

' Department of Neurology, School of Medicine, The Second Affiliated Hospital of Zhejiang University, Hangzhou, China, ${ }^{2}$ Department of Radiology, School of Medicine, The Second Affiliated Hospital of Zhejiang University, Hangzhou, China, ${ }^{3}$ The School of Medicine and Public Health, University of Newcastle, Newcastle, NSW, Australia

\section{OPEN ACCESS}

Edited by:

Cheng-Xin Gong,

New York State Institute for Basic

Research in Developmental

Disabilities, United States

Reviewed by:

Wen Hu,

New York State Office for People with Developmental Disabilities,

United States

Hui Liu,

Washington University in St. Louis,

United States

*Correspondence:

Min Lou

loumingxc@vip.sina.com

Received: 05 April 2017 Accepted: 24 May 2017

Published: 12 June 2017

Citation:

Zhong G, Zhang R, Jiaerken $Y, Y u X$,

Zhou Y, Liu C, Lin L, Tong L and

Lou M (2017) Better Correlation of Cognitive Function to White Matter Integrity than to Blood Supply

in Subjects with Leukoaraiosis.

Front. Aging Neurosci. 9:185,

doi: 10.3389/fnagi.2017.00185
Leukoaraiosis is associated with increased risk of cognitive impairment, but its pathophysiological pathway is unclear. The aim of the present study was to determine whether brain structural damage or cerebral blood supply better correlated with the global cognitive outcome in subjects with leukoaraiosis. Seventy-five subjects with leukoaraiosis were included in present study, with age ranged from 43 to 85 years, with mean white matter hyperintensities $(\mathrm{WMH})$ volume $30.69 \pm 24.35 \mathrm{~mL}$. Among them, 19(25.33\%) subjects presented with cerebral microbleeds (CMB) and 40 (53.33\%) subjects presented with lacunes. These participants received arterial spin labeling perfusion MRI, diffusion-tensor imaging (DTI) and diffusion Kurtosis imaging. We analyzed the cerebral blood flow (CBF) by dividing the brain tissue into three regions: $\mathrm{WMH}$, normal appearing white matter (NAWM) and cortex. After adjusting for age and gender, the CBF of NAWM was significantly correlated with fractional anisotropy (FA) $(r=0.336, p=0.004)$ and mean diffusion (MD) $(r=-0.271, p=0.020)$ of NAWM, while there lacked of association between CBF of cortex and mean kurtosis (MK) of cortex $(r=-0.015, p=0.912)$. Meanwhile, both NAWM-FA $(r=-0.443$, $p<0.001)$ and NAWM-MD $(r=0.293, p=0.012)$, as well as cortex-MK $(r=-0.341$, $p=0.012$ ) was significantly correlated with $\mathrm{WMH}$ volume. Univariate regression analysis demonstrated that global cognitive function was significantly associated with mean FA or $\mathrm{MD}$ of both WMH and NAWM, and cortex-CBF, but neither with the cortex-MK, nor the presences of $\mathrm{CMB}$ or lacunes. Finally, multiple linear regression analysis revealed that global cognitive function was independently associated with NAWM-FA (standardized $\beta=0.403, p<0.001$ ) and WMH-FA (Standardized $\beta=0.211, p=0.017$ ), but not with the cortex-CBF. A model that contained NAWM-FA, WMH-FA and years of education explained $49 \%$ of the variance of global cognitive function. Cerebral perfusion status might have a significant impact on the maintenance of white matter integrity in subjects with leukoaraiosis. Global cognitive function was more strongly associated with white matter integrity than with blood supply. DTI parameters, especially FA could serve as a potent imaging indicator for detecting the invisible alteration of white matter integrity and implying its potential cognitive relevance.

Keywords: cerebral blood flow, cognitive function, diffusion tensor imaging, diffusion kurtosis imaging, white matter disease 


\section{INTRODUCTION}

Leukoaraiosis, also known as white matter hyperintensities (WMH) of presumed vascular origin, are frequently seen on magnetic resonance imaging (MRI) scans of the brain in the elderly (Wardlaw et al., 2013). WMH has been viewed as an etiology of cognitive impairment and vascular dementia (VaD) (Prins and Scheltens, 2015). However, the association between the burden of WMH and severity of cognitive impairment is controversial. Some studies found that neuropsychological impairment was correlated with cerebral hypoperfusion but not the severity of WMH in patients with small vessel diseases (SVD) (Sabri et al., 1999). Evidence also showed that severe carotid stenosis with hemodynamic impairment would significantly increase the risk of cognitive deterioration in asymptomatic subjects (Balestrini et al., 2013).

The adverse connection between cerebral hypoperfusion and cognitive dysfunction may be mediated by disruption of white matter microstructural integrity, which was found to be associated with cognitive performance based on diffusion tensor imaging (DTI) studies (Nitkunan et al., 2008; Vernooij et al., 2009). Alternatively, cerebral hypoperfusion may directly compromise the brain function by down-regulation of neural metabolism and electrical activity, leading to neuronal adaptive responses (Banerjee et al., 2016; Bordeleau et al., 2016). Besides, cerebral hypoperfusion would accelerate deposition of $A \beta$-amyloid, contributing to the concurrence of $\mathrm{VaD}$ and Alzheimer's disease (AD) (Zhiyou et al., 2009). Furthermore, recent studies also suggested that cortex alterations along the course of WMH may also contribute to cognitive decline (Peres et al., 2016). Cortex alterations (e.g., Microinfarcts, atrophy) and WMH may share common underlying mechanism such as chronic hypoperfusion. The emerging technique of diffusion kurtosis imaging (DKI) is promising to detect the disruption of cortex microarchitecture and its cognitive relevance as shown in study among multiple sclerosis (MS) patients (Bester et al., 2015).

Currently, it is unclear whether brain structural damage or blood supply condition could better predict the global cognitive outcome in subjects with leukoaraiosis. In present study, we utilized multi-model MRI to assess the cerebral perfusion, white matter and cortex microstructural integrity, as well as the burden of $\mathrm{WMH}$, brain volume, presence of cerebral microbleeds (CMB) and lacunes. Then we investigated their relationship with global cognitive function among a cohort of subjects with leukoaraiosis. It is meaningful to identify potent imaging indicators for global cognitive performance, which would not only favor elucidating the pathophysiological pathway of cognitive impairment, but may also be helpful for assessment of therapeutic efficacy in future interventional trials.

\section{MATERIALS AND METHODS}

\section{Study Subjects}

This was an investigator-initiated prospective single-center study. Patients with WMH were recruited between January 2014 and April 2016 from our department. We then enrolled those who met all of the following inclusion and none of the exclusion criteria into this study. Inclusion criteria: (1) WMH visible on Fluid-Attenuated-Inversion-Recovery (FLAIR) images; (2) age $\geq 40$; (3) without a history of MS, AD, Parkinson's disease and head trauma; (4) agreed to give written informed consent. Exclusion criteria: (1) patients with secondary causes of white matter lesions, such as immunological, demyelinating, metabolic, toxic, infectious, and other established causes; (2) patients with abnormal brain MRI findings such as spaceoccupying lesions, head trauma, hemorrhage, or infarction (except for lacunar infarction); (3) patients with poor image quality. The patients admitted for TIA or acute lacunar stroke were recruited at least 3 months after the date of last stroke to avoid acute effects on MRI or neuropsychological findings. We retrieved demographic, clinical and imaging data including age, gender, years of education, and the medical history such as hypertension, diabetes mellitus, hyperlipidemia, and smoking history. Global cognitive function was assessed by Mini-Mental State Examination (MMSE).

\section{Ethics Statement}

All subjects had given written informed consent prior to the study, and the protocols had been approved by the human ethics committee of the Second Affiliated Hospital of Zhejiang University School of Medicine. All clinical investigation has been conducted according to the principles expressed in the Declaration of Helsinki.

\section{WMH Identification and Volume Measurement}

Identification of WMH on FLAIR images was according to "neuroimaging standards for research into small vessel disease" published by Wardlaw et al. (2013). WMH volume was semiautomatically measured (details seen in Image Analysis section).

\section{Definition and Detection of Cerebral Microbleeds and Lacunes}

Cerebral microbleeds were defined as small $(\leq 10 \mathrm{~mm})$, homogeneous, round foci of low signal intensity on gradient echo images in cerebellum, brainstem, basal ganglia, white matter, or cortico-subcortical junction, differentiated from vessel flow voids and mineral depositions in the globi pallidi (Greenberg et al., 2009). Lacunes were defined as rounded or ovoid lesions, 3-15 $\mathrm{mm}$ of diameter, in the basal ganglia, internal capsule, centrum semiovale, or brainstem, of cerebrospinal fluid (CSF) signal intensity on T1-,T2-weighted imaging and FLAIR, generally with a hyperintense rim on FLAIR and no increased signal on diffusion weighted-imaging (DWI)(Wardlaw et al., 2013).

\section{MRI Protocol}

All subjects underwent multi-model MRI by a 3.0 T MR (MR750, GE Healthcare, United States) scanner using an 8-channel brain phased array coil, including high-resolution three dimensional (3D) sagittal T1-weighted imaging (T1WI), FLAIR, DTI and 3D 
arterial spin labeling (ASL) perfusion MRI. A subset of subjects also received DKI. For DTI, we performed a single shot, diffusionweighted spin echo echo-planar imaging sequence. Maximum $b$-value was $1000 \mathrm{~s} / \mathrm{mm}^{2}$ in 30 non-collinear directions; 1 volume was acquired without diffusion weighting $\left(b\right.$-value $\left.=0 \mathrm{~s} / \mathrm{mm}^{2}\right)$. Other parameters of DTI were as follows: repetition time $(\mathrm{TR}) /$ echo time $(\mathrm{TE})=8000 / 80.8 \mathrm{~ms}$, flip angle $=90^{\circ}$, slice thickness $=2 \mathrm{~mm}$ without slice gap, matrix size $=128 \times 128$, field of view $(\mathrm{FOV})=25.6 \mathrm{~cm}$. DKI was acquired using a single-shot, spin-echo planar imaging (EPI) sequence with $\mathrm{TR} / \mathrm{TE}=5000 / 95 \mathrm{~ms}$; slice thickness $=2 \mathrm{~mm}$ without slice gap; FOV: $256 \mathrm{~mm} \times 256 \mathrm{~mm}$; matrix: $128 \times 128$; and three diffusion weighting $(b)$ values $\left(0,1000\right.$, and $\left.2000 \mathrm{~s} / \mathrm{mm}^{2}\right)$, with diffusion encoding in 30 directions for every $b$-value. 3D ASL was acquired using spin-echo pulse sequence with $\mathrm{TR} / \mathrm{TE}=4611 / 10.5 \mathrm{~ms}$, inversion time $(\mathrm{TI})=1525 \mathrm{~ms}$, flip angle $=111^{\circ}$, slice thickness $=4 \mathrm{~mm}$, matrix $=128 \times 128$, FOV $=24 \mathrm{~cm}$. Highresolution 3D sagittal T1-weighted imaging (T1WI) was acquired using spoiled gradient echo sequence with $\mathrm{TR} / \mathrm{TE}=7.3 / 3.0 \mathrm{~ms}$, $\mathrm{TI}=450 \mathrm{~ms}$, flip angle $=8^{\circ}$, slice thickness $=1 \mathrm{~mm}$, matrix $=250 \times 250, \mathrm{FOV}=25 \mathrm{~cm}$. Conventional MRI sequences included FLAIR $(\mathrm{TR} / \mathrm{TE}=8400 / 152 \mathrm{~ms}$, TI $=2100 \mathrm{~ms}$, flip angle $=90^{\circ}$, slice thickness $=4 \mathrm{~mm}$ without slice gap, matrix size $=256 \times 256, \mathrm{FOV}=24 \mathrm{~cm}), \mathrm{T} 1 \mathrm{WI}(\mathrm{TR} / \mathrm{TE}=1750 / 25 \mathrm{~ms}$, $\mathrm{TI}=780 \mathrm{~ms}$, flip angle $=90^{\circ}$, slice thickness $=4 \mathrm{~mm}$ without slice gap, matrix size $=256 \times 256$, FOV $=24 \mathrm{~cm})$ and DWI $\left(\mathrm{TR} / \mathrm{TE}=5000 / 86 \mathrm{~ms}\right.$, flip angle $=90^{\circ}$, slice thickness $=4 \mathrm{~mm}$ without slice gap, matrix size $=256 \times 256, \mathrm{FOV}=24 \mathrm{~cm}$, $b$-value $=1000 \mathrm{~s} / \mathrm{mm}^{2}$ along three orthogonal directions).

\section{Image Analysis}

DTI images were post-processed using $\mathrm{FSL}^{1}$ to extract brain, remove bulk motion, and eddy current induced distortions. Then we calculated the parametric maps of fractional anisotropy (FA) and mean diffusivity (MD) with DTIfit command in FSL. DKI images were corrected for brain distortion using the "eddy-current" toolbox in FSL. With Diffusion Kurtosis Estimator $(\mathrm{DKE})^{2}$, mean kurtosis $(\mathrm{MK})$ was calculated on DKI parametric map. The segmentation of gray matter, normal appearing white matter (NAWM) and WMH tissue masks was automatically processed in the native space using $3 \mathrm{D}$ T1WI and FLAIR images by lesion segmentation tool (LST) toolbox (Schmidt et al., 2012) in Statistical Parametric Mapping Version 8 (SPM8). The processed WMH, NAWM and gray matter masks were further manually corrected by an experienced neuroradiologist using ITK-SANP software ${ }^{3}$. The steps of manual correction include: (1) removal of non-brain tissue, deep gray matter, brain stem and cerebellum; (2) correction of false segmentation (positives or negatives). After coregistration, the masks of WMH, NAWM and cortex were used to obtain averaged FA, MD, MK and cerebral blood flow (CBF) of corresponding tissues in each participant (as illustrated in Supplementary Figure S1). Total brain volume was calculated

\footnotetext{
${ }^{1}$ http://www.fmrib.ox.ac.uk/fsl

${ }^{2}$ https://www.nitrc.org/projects/dke/

${ }^{3}$ www.itksnap.org
}

by taking the sum of the gray matter and white matter probabilistic tissue maps and multiplying this by the voxel volume $\left(1 \mathrm{~mm}^{3}\right)$. Intracranial volume (ICV) was calculated as the sum of total brain volume and CSF. WMH volume and brain volume be used in present study were the adjusted volume by ICV.

\section{Statistical Analysis}

All numeric variables were expressed as mean $\pm \mathrm{SD}$. The difference between $\mathrm{FA}, \mathrm{MD}$, and $\mathrm{CBF}$ of $\mathrm{WMH}$ and their counterparts of NAWM were compared using paired $t$-tests. The Pearson's partial correlation between CBF and the metrics of microstructural integrity (FA, $\mathrm{MD}$ or $\mathrm{MK}$ ) were tested, controlling for age and sex. The association of CBF, microstructural integrity, WMH volume, presences of $\mathrm{CMB}$ or lacunes with MMSE scores were tested using univariate linear regression model. The association of the variables, whose $P<0.1$ in above univariate linear regression models, with MMSE score was estimated using the multiple stepwise linear regression model (Model 1). Model 2 included the original variables of model 1, besides age, gender, years of education, brain volume, $\mathrm{WMH}$ volume, and presence of $\mathrm{CMB}$ or lacunes. A level of $P<0.05$ was considered statistically significant. All statistical analyses were performed by IBM SPSS Statistics 21 .

\section{RESULTS}

\section{Patient Characteristics}

The demographic, clinical and imaging characteristics were demonstrated in Table 1. Seventy five subjects with leukoaraiosis were included in present study. The main reasons for admission of those patients were TIA or lacunar ischemic stroke $(n=44$, $58.67 \%)$, dizziness $(n=19,25.33 \%)$, cognitive impairment $(n=5$, $6.67 \%)$, headache $(n=5,6.67 \%)$, or gait disturbance $(n=2$, $2.67 \%)$. Their age ranged from 43 to 85 years and WMH volume ranged from 1.54 to $120.51 \mathrm{~mL}$, with mean MMSE Score

TABLE 1 | Baseline characteristics.

\begin{tabular}{lc}
\hline Variable & $\boldsymbol{N}=\mathbf{7 5}$ (mean $\pm \mathbf{S D}$ ) or $\boldsymbol{n}(\mathbf{\%})$ \\
\hline Age $(y)$ & $67.05 \pm 9.62$ \\
Female\% & $39(52.00 \%)$ \\
Years of education $(y)$ & $6.44 \pm 4.75$ \\
MMSE score & $25.91 \pm 3.50$ \\
Hypertension & $52(69.33 \%)$ \\
Diabetes mellitus & $17(22.67 \%)$ \\
Hyperlipidemia & $19(25.33 \%)$ \\
Smoking & $16(21.33 \%)$ \\
WMH volume, mL & $30.69 \pm 24.35$ \\
Brain volume, mL & $1004.14 \pm 40.90$ \\
Presence of microbleeds & $19(25.33 \%)$ \\
Presence of lacunes & $40(53.33 \%)$
\end{tabular}

MMSE, Mini-Mental State Examination; FA, fractional anisotropy; MD, mean diffusivity; CBF, cerebral blood flow; WMH, white matter hyperintensities; NAWM, normal-appearing white matter. 

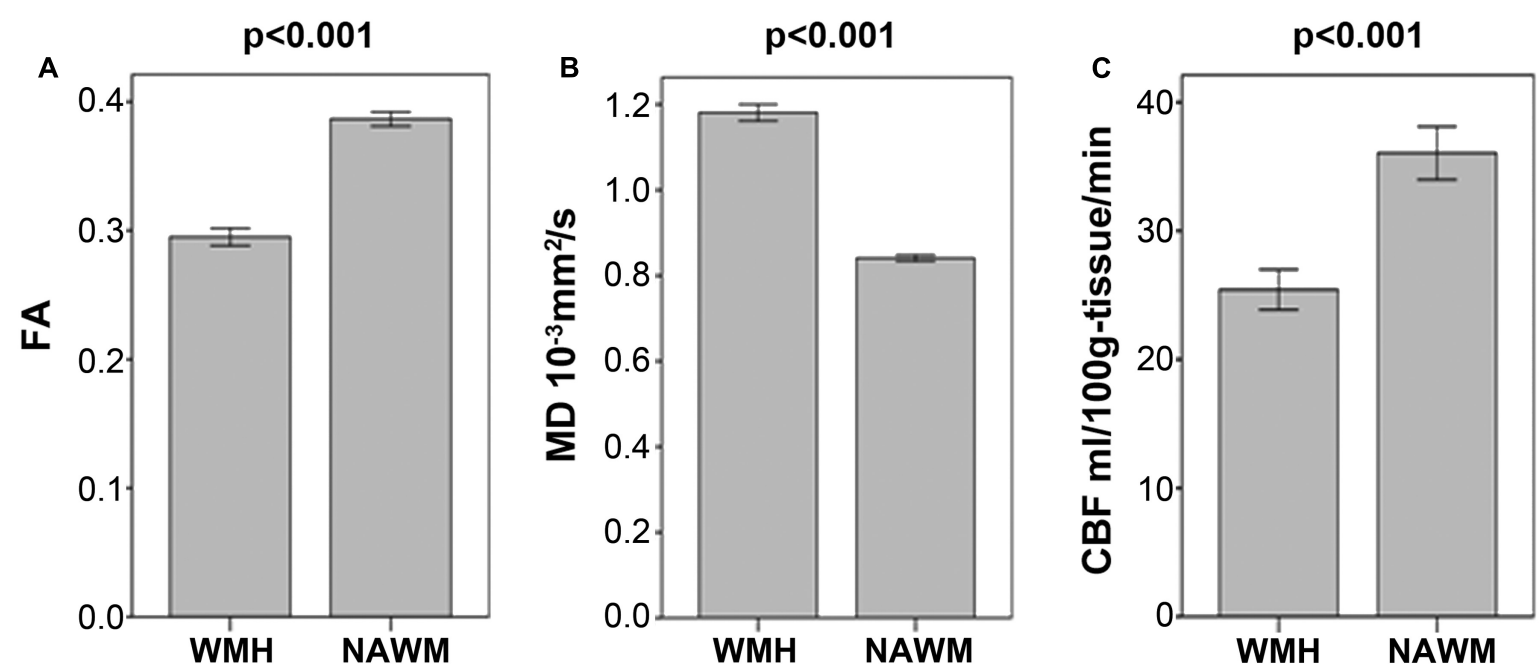

FIGURE 1 | (A-C) Comparisons of mean fractional anisotropy (FA), mean diffusion (MD) and cerebral blood flow (CBF) in white matter hyperintensities (WMH) and in their corresponding normal appearing white matter (NAWM).

$25.91 \pm 3.50$. Among them, 19(25.33\%) subjects presented with microbleeds and $40(53.33 \%)$ subjects presented with lacunes. To be note, the analysis referring to MK was restricted to a subset of 56 participants receiving DKI.

\section{Correlation between Cerebral Blood Flow and Microstructural Integrity}

As illustrated in Figure 1 and Table 2, FA and CBF of WMH were significantly decreased compared to that of NAWM, while MD was significantly increased. After adjusting for age and gender, NAWM-FA $(r=0.336, p=0.004)$ and NAWM-MD $(r=-0.271$, $p=0.020)$ were low and significantly correlated with NAWM$\mathrm{CBF}$, while there lacked of association between cortex-CBF and cortex-MK $(r=-0.015, p=0.912)$ (Table 3). Meanwhile, both NAWM-FA $(r=-0.443, p<0.001)$ and NAWM-MD $(r=0.293$, $p=0.012)$, as well as cortex-MK $(r=-0.341, p=0.012)$ were low while significantly correlated with $\mathrm{WMH}$ volume after adjusting for age and gender.

TABLE 2 | The distribution of microstructural integrity and cerebral blood flow of WMH, NAWM and Cortex.

\begin{tabular}{lc}
\hline Variable & $\boldsymbol{N}=\mathbf{7 5}$ (mean $\pm \mathbf{S D}$ ) \\
\hline WMH-FA & $0.2949 \pm 0.0292$ \\
NAWM-FA & $0.3865 \pm 0.0240$ \\
WMH-MD, $10^{-3} \mathrm{~mm}^{2} / \mathrm{s}$ & $1.1809 \pm 0.0830$ \\
NAWM-MD, $10^{-3} \mathrm{~mm}^{2} / \mathrm{s}$ & $0.8408 \pm 0.0326$ \\
WMH-CBF,mL/100g-tissue/min & $25.43 \pm 6.79$ \\
NAWM-CBF, mL/100g-tissue/min & $36.05 \pm 8.97$ \\
Cortex-MK & $0.5430 \pm 0.0982^{\#}$ \\
Cortex-CBF, mL/100g-tissue/min & $44.87 \pm 11.48$
\end{tabular}

FA, fractional anisotropy; $M D$, mean diffusivity; $C B F$, cerebral blood flow; WMH, white matter hyperintensities; NAWM, normal-appearing white matter; MK, mean kurtosis. "indicates subset analysis.
TABLE 3 | Pearson's partial correlation between cerebral blood flow and microstructural integrity after adjustment for age and sex.

\begin{tabular}{|c|c|c|c|c|c|c|}
\hline & \multicolumn{2}{|c|}{ WMH-CBF } & \multicolumn{2}{|c|}{ NAWM-CBF } & \multicolumn{2}{|c|}{ Cortex-CBF } \\
\hline & $r$ & $P$-value & $r$ & $P$-value & $r$ & $P$-value \\
\hline WMH-FA & 0.083 & 0.484 & - & - & - & - \\
\hline WMH-MD & -0.133 & 0.264 & - & - & - & - \\
\hline NAWM-FA & - & - & 0.336 & 0.004 & - & - \\
\hline NAWM-MD & - & - & -0.271 & 0.020 & - & - \\
\hline Cortex-MK & - & - & - & - & -0.015 & $0.912^{\#}$ \\
\hline
\end{tabular}

FA, fractional anisotropy; MD, mean diffusivity; CBF, cerebral blood flow; WMH, white matter hyperintensities; NAWM, normal-appearing white matter; MK, mean kurtosis. "indicates subset analysis.

\section{Relationship between Metrics of Brain Structural Damage, CBF and Global Cognitive Function}

Univariate linear regression analysis demonstrated that MMSE score was significantly associated with mean FA or MD of both WMH and NAWM, and CBF of cortex, but not with the MK of cortex (Table 4). Nevertheless, MMSE score was not significantly associated with WMH volume (standardized $\beta=-0.125, p=0.284$ ), nor the presences of $\mathrm{CMB}$ (standardized $\beta=0.060, p=0.611$ ) or presences of lacunes (standardized $\beta=-0.048, p=0.082$ ). Correlation between MMSE score and individual imaging parameters were presented in the scatter plots of Supplementary Figure S2. Finally, multiple linear regression analysis revealed that global cognitive function was independently associated with NAWMFA (standardized $\beta=0.403, p<0.001$ ) and WMH-FA (standardized $\beta=0.211, p=0.017$ ), but not with cortex-CBF (Table 5). A model that contained of NAWM-FA, WMH-FA and years of education explained $49 \%$ of the variance of MMSE score. 
TABLE 4 | The association of microstructural integrity, cerebral blood flow with MMSE score using univariate linear regression model.

\begin{tabular}{lccr}
\hline Variable & Standardized $\boldsymbol{\beta}$ & $\boldsymbol{R}^{\mathbf{2}}$ & $\boldsymbol{P}$-value \\
\hline WMH-FA & 0.328 & 0.108 & 0.004 \\
NAWM-FA & 0.501 & 0.251 & $<0.001$ \\
WMH-MD & -0.205 & 0.042 & 0.078 \\
NAWM-MD & -0.258 & 0.066 & 0.026 \\
WMH-CBF & 0.184 & 0.034 & 0.114 \\
NAWM-CBF & 0.207 & 0.043 & 0.075 \\
Cortex-CBF & 0.248 & 0.062 & 0.032 \\
Cortex-MK & 0.076 & 0.006 & $0.580^{\#}$
\end{tabular}

MMSE, Mini-Mental State Examination; FA, fractional anisotropy; MD, mean diffusivity; CBF, cerebral blood flow; WMH, white matter hyperintensities. NAWM, normal-appearing white matter; $M K$, mean kurtosis. $R^{2}$ represents the proportion of variation in MMSE score explained by individual variable. "indicates subset analysis.

TABLE 5 | The association of relevant variables * with MMSE score using multiple stepwise linear regression model.

\begin{tabular}{lcrc}
\hline & Standardized $\boldsymbol{\beta}$ & $\boldsymbol{P}$-value & $\boldsymbol{R}^{\mathbf{2}}$ \\
\hline Model 1 & & & \\
WMH-FA & 0.266 & 0.008 & 0.321 \\
NAWM-FA & 0.466 & $<0.001$ & \\
Model 2 & & & 0.490 \\
WMH-FA & 0.211 & 0.017 & \\
NAWM-FA & 0.403 & $<0.001$ & \\
years of education & 0.421 & $<0.001$ & \\
\hline
\end{tabular}

FA, fractional anisotropy; WMH, white matter hyperintensities; NAWM, normalappearing white matter. * Relevant variables included WMH-FA, NAWM-FA, WMH$M D$, NAWM-MD, NAWM-CBF and cortex-CBF in model 1; Additionally, age, gender, years of education, brain volume, WMH volume, and presence of cerebral microbleeds or lacunes were further included in Model 2. $R^{2}$ represents the proportion of variation in MMSE score explained by all variables in model 1 and model 2.

\section{DISCUSSION}

For subjects with leukoaraiosis, brain structural damage has been reported to cause their cognitive impairment, whereas evidence also showed the importance of blood supply in affecting their cognitive outcome. Limited studies have been conducted to link the two underlying mechanisms. This study explored the relationship of these two mechanisms and results showed that global cognitive function was more strongly associated with white matter integrity than with blood supply in subjects with leukoaraiosis.

Degradation of the white matter microarchitecture could be detected by changes in measurable DTI parameters, reflecting as decreased FA or increased MD (Moseley, 2002). FA and MD of NAWM were found to be significantly associated with $\mathrm{WMH}$ volume in present study, which implied that invisible structural damages were beyond the boundary of WMH lesion. And we observed that FA of both WMH and NAWM were closely correlated with global cognitive performance. And a model that contained FA of WMH, FA of NAWM and years of education could explain nearly half of the variance of MMSE score. This was consistent with previous large population-based study which demonstrated that DTI parameters of both WMH and NAWM were significantly associated with cognitive function, regardless of WMH load and brain atrophy (Vernooij et al., 2009). Study conducted by Nitkunan et al. (2008) also demonstrated that the variance of executive function could largely be explained by a model that contained peak height of FA, brain volume, together with age, gender, and premorbid IQ among 35 patients with SVD.

Meanwhile, our data again showed weak correlation between WMH load and cognitive performance, which could be attributed to the difference of underlying pathological changes both in similar appearing WMH lesions and in NAWM. Postmortem studies also proved that WMH are heterogeneous in terms of histopathology, ranging from slight disentanglement of the matrix to varying degrees of demyelination and axonal loss (Gouw et al., 2011).

Previous study reported that neuropsychological impairment was significantly correlated with cerebral hypoperfusion but not the severity of WMH in patients with SVD (Sabri et al., 1999). Moser et al.'s (2012) study also found that global CBF were significantly associated with global neuropsychological functioning after adjusting for age and sex. Nevertheless, these studies did not take into account the invisible alterations of white mater integrity, which was significantly correlated with the cognitive function in present and other studies. We posited that the previous reported adverse connection between cerebral hypoperfusion and cognitive dysfunction might be mediated by disruption of white matter integrity secondary to chronic hypoperfusion. Supporting this assumption, we found that $\mathrm{CBF}$ of WMH was significantly decreased compared to that of NAWM, and CBF of NAWM was significantly correlated with FA of NAWM after adjusting for age and sex. Another study based on PET-CT also showed no association between baseline global CBF and MMSE score among 27 cognitively intact individuals with hypertension and WMH (Kitagawa et al., 2009). However, this study prospectively observed that baseline CBF was linearly associated with cognitive decline assessed by MMSE score over a 3-years period. This paradoxical finding could be explained by that lower baseline CBF leads to accelerated but non-proportionate disruption of white matter integrity, which then contributes to cognitive deterioration.

Diffusion kurtosis imaging allows the measurement of $\mathrm{MK}$, which does not require tissue's directionality and hence it is applicable for measuring microstructural integrity of cortex. Previous studies have shown that cortical atrophy was associated with the burden of $\mathrm{WMH}$, but little is known about the condition of the tissue not yet lost. We found that cortical MK was inversely associated with $\mathrm{WMH}$ volume, while there lacked of association between cortical MK and cortical CBF. This could be pathophysiologically explained by that cortex alterations may be secondary to remote white matter degeneration (e.g., WMH) other than hypoperfusion. Among 34 MS patients, a preliminary study found that decreased MK of cortex was correlated with poor cognitive performance (Bester et al., 2015). By contrast, we found no significant association between $\mathrm{MK}$ of cortex and cognitive function, indicating the relative health of the existing cortex with regard to neuronal degeneration. However, the exact 
pathological substrate responsible for MK changes of cortex has yet to be elucidated.

To the best of our knowledge, few previous work, if any, has investigated the correlation between cerebral perfusion and brain microstructural integrity, as well as their relationship with global cognitive function in subjects with leukoaraiosis. Methodologically, we analyzed the CBF by dividing the brain tissue into three regions: WMH, NAWM and cortex. By contrast, previous studies based on PET-CT could only measure the $\mathrm{CBF}$ of regional lobe or whole brain due to its limited spatial resolution. Given the $\mathrm{CBF}$ varies greatly in white matter and cortex, mixed quantification of $\mathrm{CBF}$ in these territories would compromise its reliability in assessing its relationship with structural metrics and cognitive function. Our findings suggested that DTI parameters, especially FA of NAWM, could serve as a candidate imaging indicator for monitoring alterations of global white matter integrity and implying its cognitive relevance. By contrast, cerebral blood supply is not an ideal indicator for predicting cognitive function in subjects with leukoaraiosis. Though cerebral hypoperfusion has detrimental effect on the integrity of white matter, ischemic tolerance may vary among different individuals. Besides, risk factors other than hypoperfusion may also contribute to the injury of white matter, such as endothelial dysfunction and increased blood brain barrier permeability (Wardlaw, 2010).

Our study also has some limitations. Firstly, the modest sample size may limit its power to reveal the relationship between cognitive function and imaging parameters. Secondly, our study design was cross-sectional and the main conclusions were made from correlational analyses. Therefore, longitudinal studies with larger sample size should be more reward to explore the causality between cerebral blood supply, brain structural damage and cognitive outcome. Thirdly, we could not make sure all the AD patients have been precluded. In fact, combined involvement of AD-type and vascular pathology were commonly seen in elderly people with cognitive decline (Neuropathology Group. Medical Research Council Cognitive Function and Aging Study, 2001). Lastly, the global cognitive function was merely assessed by most extensively used MMSE score, which may not be sensitive enough for detecting mild cognitive impairment.

\section{CONCLUSION}

Cerebral perfusion status may have a significant impact on the maintenance of white matter health in subjects with leukoaraiosis. Meanwhile, cortex alterations along with $\mathrm{WMH}$ may mainly secondary to remote white matter degeneration other than hypoperfusion. Global cognitive outcome was more strongly associated with white matter integrity than with blood supply. DTI parameters, especially FA could serve as a potent

\section{REFERENCES}

Balestrini, S., Perozzi, C., Altamura, C., Vernieri, F., Luzzi, S., Bartolini, M., et al. (2013). Severe carotid stenosis and impaired cerebral hemodynamics can imaging indicator for detecting the invisible disruption of white matter microarchitecture and implying its potential cognitive sequelae.

\section{AUTHOR CONTRIBUTIONS}

GZ drafted and revised the manuscript, participated in study concept and design, conducted the statistical analyses, analyzed and interpreted the data. ML participated in study concept and design, data interpretation and made a major contribution in revising the manuscript. $\mathrm{RZ}$ participated in the study design and made contribution in revising the manuscript. YJ, XY, and $\mathrm{YZ}$ assisted in designing the MRI sequences and imaging analysis. CL assessed the cognitive function of the participants. LT made contribution in revising the manuscript. LL validated the statistical analyses and made contribution in revising the manuscript.

\section{FUNDING}

The author's disclosed receipt of the following financial support for the research, authorship, and/or publication of this article: This study was supported by grants from National Natural Science Foundation of China (81471170, 81622017).

\section{ACKNOWLEDGMENTS}

Thanks to the guidance of statistical analysis from Yunxian Yu, an associate professor from the department of epidemiology and health statistics, School of Public Health, Zhejiang University, Hangzhou, China.

\section{SUPPLEMENTARY MATERIAL}

The Supplementary Material for this article can be found online at: http://journal.frontiersin.org/article/10.3389/fnagi. 2017.00185/full\#supplementary-material

FIGURE S1 | Multimodal MRI images from a typical subject with leukoaraiosis: (A) FLAIR image; (B) WMH mask (red); (C) NAWM mask (red); (D) Cortex mask (red); (E-H) were the co-registered corresponding FA, MD, MK and CBF images, respectively. FLAIR, fluid attenuated inversion recovery; $\mathrm{WMH}$, white matter hyperintensities; NAWM, normal-appearing white matter; FA, fractional anisotropy. $\mathrm{MD}$, mean diffusivity; MK, mean kurtosis; CBF, cerebral blood flow.

FIGURE S2 | (A-H) Scatter plots of mini-mental state examination (MMSE) score in relations to WMH-FA (A), NAWM-FA (B), WMH-MD (C), NAWM-MD (D), WMH-CBF (E), NAWM-CBF (F), Cortex-CBF (G), and Cortex-MK\# (H), respectively. "Indicates subset analysis.

influence cognitive deterioration. Neurology 80, 2145-2150. doi: 10.1212/WNL. 0b013e318295d71a

Banerjee, G., Wilson, D., Jager, H. R., and Werring, D. J. (2016). Novel imaging techniques in cerebral small vessel diseases and vascular cognitive 
impairment. Biochim. Biophys. Acta 1862, 926-938. doi: 10.1016/j.bbadis.2015. 12.010

Bester, M., Jensen, J. H., Babb, J. S., Tabesh, A., Miles, L., Herbert, J., et al. (2015). Non-Gaussian diffusion MRI of gray matter is associated with cognitive impairment in multiple sclerosis. Multiple Scler. J. 21, 935-944. doi: 10.1177/ 1352458514556295

Bordeleau, M., ElAli, A., and Rivest, S. (2016). Severe chronic cerebral hypoperfusion induces microglial dysfunction leading to memory loss in APPswe/PS1 mice. Oncotarget 7, 11864-11880. doi: 10.18632/oncotarget.7689

Gouw, A. A., Seewann, A., van der Flier, W. M., Barkhof, F., Rozemuller, A. M., Scheltens, P., et al. (2011). Heterogeneity of small vessel disease: a systematic review of MRI and histopathology correlations. J. Neurol. Neurosurg. Psychiatry 82, 126-135. doi: 10.1136/jnnp.2009.204685

Greenberg, S. M., Vernooij, M. W., Cordonnier, C., Viswanathan, A., Al-Shahi Salman, R., Warach, S., et al. (2009). Cerebral microbleeds: a guide to detection and interpretation. Lancet Neurol. 8, 165-174. doi: 10.1016/S1474-4422(09) 70013-4

Kitagawa, K., Oku, N., Kimura, Y., Yagita, Y., Sakaguchi, M., Hatazawa, J., et al. (2009). Relationship between cerebral blood flow and later cognitive decline in hypertensive patients with cerebral small vessel disease. Hypertens. Res. 32, 816-820. doi: 10.1038/hr.2009.100

Moseley, M. (2002). Diffusion tensor imaging and aging - a review. NMR Biomed. $15,553-560$.

Moser, D. J., Boles Ponto, L. L., Miller, I. N., Schultz, S. K., Menda, Y., Arndt, S., et al. (2012). Cerebral blood flow and neuropsychological functioning in elderly vascular disease patients. J. Clin. Exp. Neuropsychol. 34, 220-225. doi: 10.1080/ 13803395.2011.630653

Neuropathology Group. Medical Research Council Cognitive Function and Aging Study. (2001). Pathological correlates of late-onset dementia in a multicentre, community-based population in England and Wales. Neuropathology Group of the Medical Research Council Cognitive Function and Ageing Study (MRC CFAS). Lancet 357, 169-175.

Nitkunan, A., Barrick, T. R., Charlton, R. A., Clark, C. A., and Markus, H. S. (2008). Multimodal MRI in cerebral small vessel disease: its relationship with cognition and sensitivity to change over time. Stroke 39, 1999-2005. doi: 10.1161/STROKEAHA.107.507475

Peres, R., De Guio, F., Chabriat, H., and Jouvent, E. (2016). Alterations of the cerebral cortex in sporadic small vessel disease: a systematic review of in vivo MRI data. J. Cereb. Blood Flow Metab. 36, 681-695. doi: 10.1177/ $0271678 X 15625352$
Prins, N. D., and Scheltens, P. (2015). White matter hyperintensities, cognitive impairment and dementia: an update. Nat. Rev. Neurol. 11, 157-165. doi: $10.1038 /$ nrneurol.2015.10

Sabri, O., Ringelstein, E. B., Hellwig, D., Schneider, R., Schreckenberger, M., Kaiser, H. J., et al. (1999). Neuropsychological impairment correlates with hypoperfusion and hypometabolism but not with severity of white matter lesions on MRI in patients with cerebral microangiopathy. Stroke 30, 556-566.

Schmidt, P., Gaser, C., Arsic, M., Buck, D., Förschler, A., Berthele, A., et al. (2012). An automated tool for detection of FLAIR-hyperintense white-matter lesions in Multiple Sclerosis. Neuroimage 59, 3774-3783. doi: 10.1016/j.neuroimage.2011. 11.032

Vernooij, M. W., Ikram, M. A., Vrooman, H. A., Wielopolski, P. A., Krestin, G. P., Hofman, A., et al. (2009). White matter microstructural integrity and cognitive function in a general elderly population. Arch. Gen. Psychiatry 66, 545-553. doi: 10.1001/archgenpsychiatry.2009.5

Wardlaw, J. M. (2010). Blood-brain barrier and cerebral small vessel disease. J. Neurol. Sci. 299, 66-71. doi: 10.1016/j.jns.2010. 08.042

Wardlaw, J. M., Smith, E. E., Biessels, G. J., Cordonnier, C., Fazekas, F., Frayne, R., et al. (2013). Neuroimaging standards for research into small vessel disease and its contribution to ageing and neurodegeneration. Lancet Neurol. 12, 822-838. doi: 10.1016/S1474-4422(13)70124-8

Zhiyou, C., Yong, Y., Shanquan, S., Jun, Z., Liangguo, H., Ling, Y., et al. (2009). Upregulation of BACE1 and beta-amyloid protein mediated by chronic cerebral hypoperfusion contributes to cognitive impairment and pathogenesis of Alzheimer's disease. Neurochem. Res. 34, 1226-1235. doi: 10.1007/s11064008-9899-y

Conflict of Interest Statement: The authors declare that the research was conducted in the absence of any commercial or financial relationships that could be construed as a potential conflict of interest.

Copyright (c) 2017 Zhong, Zhang, Jiaerken, Yu, Zhou, Liu, Lin, Tong and Lou. This is an open-access article distributed under the terms of the Creative Commons Attribution License (CC BY). The use, distribution or reproduction in other forums is permitted, provided the original author(s) or licensor are credited and that the original publication in this journal is cited, in accordance with accepted academic practice. No use, distribution or reproduction is permitted which does not comply with these terms. 